\title{
Natural Language Processing for the Identification of Silent Brain Infarcts From Neuroimaging Reports
}

Sunyang Fu ${ }^{1}$, MHI; Lester Y Leung ${ }^{2}$, MD; Yanshan Wang ${ }^{1}, \mathrm{PhD}$; Anne-Olivia Raulli ${ }^{2}, \mathrm{MD}$; David F Kallmes ${ }^{3}$, MD; Kristin A Kinsman ${ }^{3}$, MD; Kristoff B Nelson ${ }^{2}$, MD; Michael S Clark ${ }^{3}$, MD; Patrick H Luetmer ${ }^{3}$, MD; Paul R Kingsbury ${ }^{1}$, $\mathrm{PhD}$; David M Kent ${ }^{4}, \mathrm{MD}$; Hongfang $\mathrm{Liu}^{1}, \mathrm{PhD}$

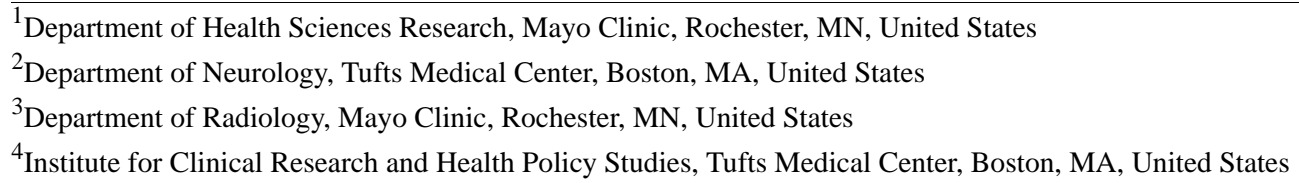

Corresponding Author:

Hongfang Liu, PhD

Department of Health Sciences Research

Mayo Clinic

205 3rd Ave SW

Rochester, MN,

United States

Phone: 15077730057

Email: Liu.Hongfang@mayo.edu

\begin{abstract}
Background: Silent brain infarction (SBI) is defined as the presence of 1 or more brain lesions, presumed to be because of vascular occlusion, found by neuroimaging (magnetic resonance imaging or computed tomography) in patients without clinical manifestations of stroke. It is more common than stroke and can be detected in $20 \%$ of healthy elderly people. Early detection of SBI may mitigate the risk of stroke by offering preventative treatment plans. Natural language processing (NLP) techniques offer an opportunity to systematically identify SBI cases from electronic health records (EHRs) by extracting, normalizing, and classifying SBI-related incidental findings interpreted by radiologists from neuroimaging reports.
\end{abstract}

Objective: This study aimed to develop NLP systems to determine individuals with incidentally discovered SBIs from neuroimaging reports at 2 sites: Mayo Clinic and Tufts Medical Center.

Methods: Both rule-based and machine learning approaches were adopted in developing the NLP system. The rule-based system was implemented using the open source NLP pipeline MedTagger, developed by Mayo Clinic. Features for rule-based systems, including significant words and patterns related to SBI, were generated using pointwise mutual information. The machine learning models adopted convolutional neural network $(\mathrm{CNN})$, random forest, support vector machine, and logistic regression. The performance of the NLP algorithm was compared with a manually created gold standard. The gold standard dataset includes 1000 radiology reports randomly retrieved from the 2 study sites (Mayo and Tufts) corresponding to patients with no prior or current diagnosis of stroke or dementia. 400 out of the 1000 reports were randomly sampled and double read to determine interannotator agreements. The gold standard dataset was equally split to 3 subsets for training, developing, and testing.

Results: Among the 400 reports selected to determine interannotator agreement, 5 reports were removed due to invalid scan types. The interannotator agreements across Mayo and Tufts neuroimaging reports were 0.87 and 0.91 , respectively. The rule-based system yielded the best performance of predicting SBI with an accuracy, sensitivity, specificity, positive predictive value (PPV), and negative predictive value (NPV) of 0.991, 0.925, 1.000, 1.000, and 0.990, respectively. The CNN achieved the best score on predicting white matter disease (WMD) with an accuracy, sensitivity, specificity, PPV, and NPV of 0.994, 0.994, 0.994, 0.994, and 0.994 , respectively.

Conclusions: We adopted a standardized data abstraction and modeling process to developed NLP techniques (rule-based and machine learning) to detect incidental SBIs and WMDs from annotated neuroimaging reports. Validation statistics suggested a high feasibility of detecting SBIs and WMDs from EHRs using NLP. 


\section{KEYWORDS}

natural language processing; neuroimaging; electronic health records

\section{Introduction}

\section{Background}

Silent brain infarction (SBI) is defined as the presence of 1 or more brain lesions, presumed to be because of vascular occlusion, found by neuroimaging (magnetic resonance imaging, MRI or computed tomography, CT) in patients without clinical manifestations of stroke. SBIs are more common than stroke and can be detected on MRI in $20 \%$ of healthy elderly [1-3]. Studies have shown that SBIs are associated with increased risk of subsequent stroke, cognitive decline, and deficiency in physical function $[1,2]$. Despite the high prevalence and serious consequences, there is no consensus on the management of SBI as routinely discovering SBIs is challenged by the absence of corresponding diagnosis codes and the lack of the knowledge about the characteristics of the affected population, treatment patterns, or the effectiveness of therapy [1]. Even though there is strong evidence shows that antiplatelet and statin therapies are effective in preventing recurrent stroke in patients with prior stroke, the degree to which these results might apply to patients with SBI is unclear. Although SBI is understood by some clinicians to be pathophysiologically identical to stroke (and thus similarly treated), others view SBI as an incidental neuroimaging finding of unclear significance. The American Heart Association/American Stroke Association has identified SBI as a major priority for new studies on stroke prevention because the population affected by SBI falls between primary and secondary stroke prevention [4].

In addition to SBI, white matter disease (WMD) or leukoaraiosis is another common finding in neuroimaging of elderly. Similar to SBI, WMD is usually detected incidentally on brain scans and is commonly believed to be a form of microvascular ischemic brain damage resulting from typical cardiovascular risk factors [5]. WMD is associated with subcortical infarcts due to small vessel disease and is predictive of functional disability, recurrent stroke, and dementia [6-8]. SBI and WMD are related, but it is unclear whether they result from the same, independent, or synergistic processes $[9,10]$. As with SBI, there are no proven preventive treatments or guidelines regarding the initiation of risk factor-modifying therapies when WMD is discovered.

\section{Objectives}

Identifying patients with SBI is challenged by the absence of corresponding diagnosis codes. One reason is that SBI-related incidental findings are not included in a patient's problem list or other structured fields of electronic health records (EHRs); instead, the findings are captured in neuroimaging reports. A neuroimaging report is a type of EHR data that contains the interpretation and finding from neuroimage such as CT and MRI in unstructured text. Incidental SBIs can be detected by the review of neuroradiology reports obtained in clinical practice, typically performed manually by radiologists or neurologists. However, manually extracting information from patient narratives is time-consuming, costly, and lacks robustness and standardization [11-14]. Natural language processing (NLP) has been leveraged to perform chart review for other medical conditions by automatically extracting important clinical concepts from unstructured text. Researchers have used NLP systems to identify clinical syndromes and biomedical concepts from clinical notes, radiology reports, and surgery operative notes [15]. An increasing amount of NLP-enabled clinical research has been reported, ranging from identifying patient safety occurrences [16] to facilitating pharmacogenomic studies [17]. Our study focuses on developing NLP algorithms to routinely detect incidental SBIs and WMDs.

\section{Methods}

\section{Study Setting}

This study was approved by the Mayo Clinic and Tufts Medical Center (TMC) institutional review boards. This work is part of the Effectiveness of Stroke PREvention in Silent StrOke project, which is to use NLP techniques to identify individuals with incidentally discovered SBIs from radiology reports, at 2 sites: Mayo Clinic and TMC.

\section{Gold Standard}

The detailed process of generating the gold standard is described in Multimedia Appendix 1. The gold standard annotation guideline was developed by 2 subject matter experts: a vascular neurologist (LYL) and a neuroradiologist (PHL), and the annotation task was performed by 2 third-year residents (KAK, MSC) from Mayo and 2 first-year residents (AOR, KN) from TMC. Each report was annotated with 1 of the 3 labels for SBI (positive SBI, indeterminate SBI, or negative SBI) and one of the 3 labels for WMD (positive WMD, indeterminate WMD, or negative WMD).

The gold standard dataset includes 1000 radiology reports randomly retrieved from the 2 study sites (500 from Mayo Clinic and 500 from TMC) corresponding to patients with no prior or current diagnosis of stroke or dementia. To calculate interannotator agreement (IAA), 400 out of the 1000 reports were randomly sampled and double read. The gold standard dataset was equally split to 3 subsets for training (334), developing (333), and testing (333).

\section{Experimental Methods}

We compared 2 NLP approaches. One was to define the task an information extraction (IE) task, where a rule-based IE system can be developed to extract SBI or WMD findings. The other was to define the task as a sentence classification task, where sentences can be classified to contain SBI or WMD findings.

\section{Rule-Based Information Extraction}

We adopted the open source NLP pipeline, MedTagger, as the infrastructure for the rule-based system implementation. MedTagger is a resource-driven, open source unstructured information management architecture-based IE framework [18]. The system separates task-specific NLP knowledge engineering 
from the generic NLP process, which enables words and phrases containing clinical information to be directly coded by subject matter experts. The tool has been utilized in the eMERGE consortium to develop NLP-based phenotyping algorithms [19]. Figure 1 shows the process workflow. The generic NLP process includes sentence tokenization, text segmentation, and context detection. The task-specific NLP process includes the detection of concept mentions in the text using regular expressions and normalized to specific concepts. The summarization component applies heuristic rules for assigning the labels to the document.

For example, the sentence "probable right old frontal lobe subcortical infarct as described above," is processed as an SBI concept with the corresponding contextual information with status as "probable," temporality as "present," and experiencer as "patient."

The domain-specific NLP knowledge engineering was developed following 3 steps: (1) Prototype algorithm development, (2) Formative algorithm development using the training data, and (3) Final algorithm evaluation. We leveraged pointwise mutual information [20] to identify significant words and patterns associated with each condition for prototyping the algorithm (Multimedia Appendix 2). The algorithm was applied to the training data. False classified reports were manually reviewed by 2 domain experts (LYL, PHL). Keywords were manually curated through an iteratively refining process until all issues were resolved. The full list of concepts, keywords, modifiers, and diseases categories are listed in Textbox 1.

Figure 1. Rule system process flow. SBI: silent brain infarction; WMD: white matter disease.

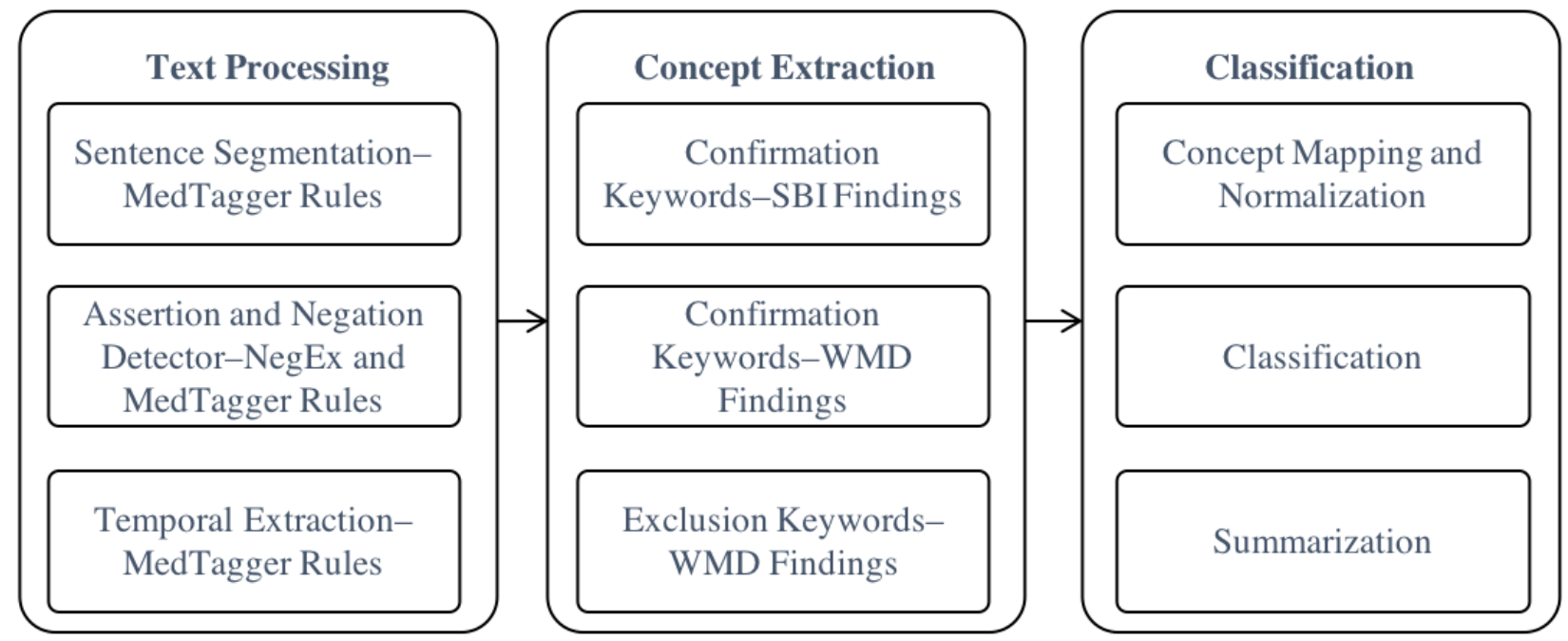

Textbox 1. Silent brain infarction (SBI) and white matter disease (WMD) risk factor and indication keywords.

- Confirmation keywords—disease-finding SBI: infarct, infarcts, infarctions, infarction, lacune, lacunes

- Confirmation keywords—disease modifier SBI: acute, acute or subacute, recent, new, remote, old, chronic, prior, chronic foci of, benign, stable small, stable

- Confirmation keywords - disease location SBI: territorial, lacunar, cerebellar, cortical, frontal, caudate, right frontoparietal lobe, right frontal cortical, right frontal lobe, embolic, left basal ganglia lacunar, basal ganglia lacunar, left caudate and left putamen lacunar

- Confirmation keywords - disease-finding WMD: leukoaraiosis, white matter, microvascular ischemic, microvascular leukemic, microvascular degenerative

- Exclusion WMD: degenerative changes

\section{Machine Learning}

The machine learning (ML) approach allows the system to automatically learn robust decision rules from labeled training data. The task was defined as a sequential sentence classification task. We adopted Kim's convolutional neural network (CNN) [21] and implemented using TensorFlow 1.1.02 [22]. The model architecture, shown in Figure 2, is a variation of the CNN architecture of Collobert R [23].

We also adopted 3 traditional ML models—random forest [24], support vector machine [25] and logistic regression [26] - for baseline comparison. All models used word vector as input representation, where each word from the input sentence is represented as the k-dimensional word vector. The word vector is generated from word embedding, a learned representation for text where words that have the same meaning have a similar representation. Suppose $\mathrm{x}_{1}, \mathrm{x}_{2}, \ldots, \mathrm{x}_{\mathrm{n}}$ is the sequence of word representations in a sentence where

$$
\mathrm{x}_{\mathrm{i}}=\mathrm{E}_{\mathrm{xi}}, \mathrm{I}=1,2, \ldots, \mathrm{n} .
$$

Here, $E_{x i}$ is the word embedding representation for word $x_{i}$ with the dimensionality d. In our ML experiment, we used Wang's word embedding trained from Mayo Clinic clinical notes where $\mathrm{d}=100$ [27]. The embedding model is the skip-gram of word2vec, an architecture proposed by Mikolov T [28]. Let 
$\mathrm{x}_{\mathrm{i}: \mathrm{i}+\mathrm{k}-1}$ represent a window of size $\mathrm{k}$ in the sentence. Then the output sequence of the convolutional layer is

$$
\operatorname{con}_{\mathrm{i}}=\mathrm{f}\left(\mathrm{w}_{\mathrm{k}} \mathrm{x}_{\mathrm{i}: \mathrm{i}+\mathrm{k}-1}+\mathrm{b}_{\mathrm{k}}\right) \text {, }
$$

where $f$ is a rectify linear unit function, $w_{k}$ and $b_{k}$ are the learning parameters. Max pooling was then performed to record the largest number from each feature map. By doing so, we obtained fixed length global features for the whole sentence, that is,

$$
\mathrm{m}_{\mathrm{k}}=\max _{1 \leq \mathrm{i} \leq \mathrm{n}-\mathrm{k}+1}\left(\operatorname{con}_{\mathrm{i}}\right) .
$$

Then the features are fit into a fully connected layer with the output being the final feature vector $\mathrm{O}=\mathrm{wm}_{\mathrm{k}}+\mathrm{b}$. Finally, a softmax function is utilized to make final classification decision, that is,

$$
\mathrm{p}(\text { sbi } \mid \mathrm{x}, \theta)=\mathrm{e}^{\wedge}\left(\mathrm{O}_{\text {sbi }}\right) /\left(\mathrm{e}^{\wedge}\left(\mathrm{O}_{\text {sbi }}\right)+\mathrm{e}^{\wedge}\left(\mathrm{O}_{\text {other }}\right)\right),
$$

where $\theta$ is a vector of the hyper parameters of the model, such as $\mathrm{w}_{\mathrm{k}}, \mathrm{b}_{\mathrm{k}}$, w and $\mathrm{b}$.

Figure 2. Convolutional neural network architecture with 2 channels for an example sentence.

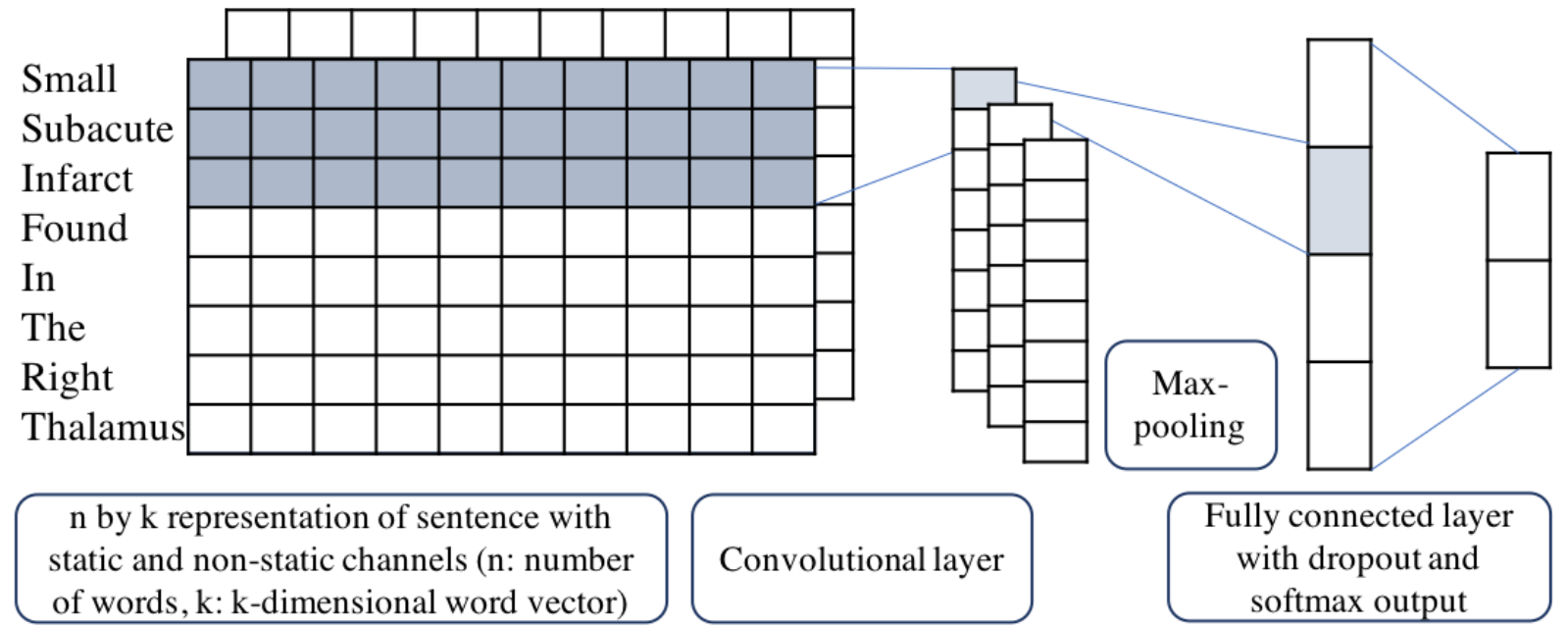

\section{Evaluation Metric}

For evaluation of the quality of the annotated corpus, Cohen kappa was calculated to measure the IAA during all phases [29]. As the primary objective of the study is case ascertainment, we calculated the IAA at the report level.

A $2 \times 2$ confusion matrix was used to calculate performance score for model evaluation: positive predictive value (PPV), sensitivity, negative predictive value (NPV), specificity, and accuracy using manual annotation as the gold standard. The McNemar test was adopted to evaluate the performance difference between the rule-based and ML models [30,31]. To have a better understanding of the potential variation between neuroimaging reports and neuroimages, we compared the model with the best performance (rule-based) with neuroimaging interpretation. A total of $12 \mathrm{CT}$ images and $12 \mathrm{MRI}$ images were stratified-randomly sampled from the test set. A total of 2 attending neurologists read all 24 images and assigned the SBI and WMD status. The cases with discrepancies were adjudicated by the neuroradiologist (PHL) The agreement was assessed using kappa and F-measure [32].

\section{Results}

\section{Interannotator Agreements Across Neuroimaging Reports}

Among the total 400 double-read reports, 5 reports were removed because of invalid scan types. The IAAs across Mayo and Tufts neuroimaging reports were 0.87 and 0.91 . Overall, there is a high agreement between readers on both reports (Tables 1 and 2). Age-specific prevalence of SBI and WMD is

\begin{tabular}{|c|c|c|c|c|c|c|}
\hline \multirow[t]{2}{*}{ Interannotator agreement } & \multicolumn{2}{|c|}{ Computed tomography $(n=63)$} & \multicolumn{2}{|c|}{ Magnetic resonance imaging $(n=144)$} & \multicolumn{2}{|c|}{ Total $(n=207)$} \\
\hline & $\%$ agree & kappa & $\%$ agree & kappa & $\%$ agree & kappa \\
\hline Silent brain infarction & 98.4 & 0.92 & 97.2 & 0.83 & 97.6 & 0.87 \\
\hline White matter disease & 100.0 & 1.00 & 98.6 & 0.97 & 99.0 & 0.98 \\
\hline
\end{tabular}
provided in Multimedia Appendix 2.

Table 1. Interreader agreement across 207 Mayo neuroimaging reports. 
Table 2. Interreader agreement across 188 Tufts Medical Center neuroimaging reports.

\begin{tabular}{|c|c|c|c|c|c|c|}
\hline \multirow[t]{2}{*}{ Interannotator agreement } & \multicolumn{2}{|c|}{ Computed tomography $(\mathrm{n}=80)$} & \multicolumn{2}{|c|}{ Magnetic resonance imaging (108) } & \multicolumn{2}{|c|}{ Total $(n=188)$} \\
\hline & $\%$ agree & kappa & $\%$ agree & kappa & $\%$ agree & kappa \\
\hline Silent brain infarction & 98.8 & 0.79 & 99.1 & 0.94 & 99.5 & 0.91 \\
\hline White matter disease & 100.0 & 1.00 & 99.1 & 0.98 & 99.5 & 0.99 \\
\hline
\end{tabular}

\section{Natural Language Processing System Performance}

Overall, the rule-based system yielded the best performance of predicting SBI with an accuracy of 0.991. The CNN achieved the best score on predicting WMD (0.994). Full results are provided in Table 3.

According to the McNemar test, we found the difference between rule-based system and CNN on SBI is considered to be statistically significant $(P$ value $=.03)$. We found no statistically significant difference between the rest of the models.
Table 4 lists the evaluation results of NLP and gold standard derived from reports against the neuroimaging interpretation for SBI and WMD. Both NLP and gold standard had moderate-high agreements with the neuroimaging interpretation, with kappa scores around .5. Our further analysis showed the practice graded findings (gold standard and NLP) achieved high precision and moderate recall scores compared with the neuroimaging interpretation. Through the confirmation with Mayo and TMC radiologists, we believed such discrepancy was because of the inconsistency in documentation standards related to clinical incidental findings, causing SBIs and WMDs underreported.

Table 3. Performance on test dataset against human annotation as gold standard.

\begin{tabular}{|c|c|c|c|c|c|}
\hline $\begin{array}{l}\text { Evaluation of natural language processing, } \\
\text { model name }\end{array}$ & Sensitivity & Specificity & $\begin{array}{l}\text { Positive predictive } \\
\text { value }\end{array}$ & $\begin{array}{l}\text { Negative predictive } \\
\text { value }\end{array}$ & Accuracy \\
\hline \multicolumn{6}{|l|}{ Silent brain infarction $(\mathrm{n}=\mathbf{3 3 3})$} \\
\hline Rule-based system & 0.925 & 1.000 & 1.000 & 0.990 & 0.991 \\
\hline $\mathrm{CNN}^{\mathrm{a}}$ & 0.650 & 0.993 & 0.929 & 0.954 & 0.952 \\
\hline Logistic regression & 0.775 & 0.983 & 0.861 & 0.970 & 0.958 \\
\hline $\mathrm{SVM}^{\mathrm{b}}$ & 0.825 & 1.000 & 1.000 & 0.977 & 0.979 \\
\hline Random forest & 0.875 & 1.000 & 1.000 & 0.983 & 0.986 \\
\hline \multicolumn{6}{|l|}{ White matter disease $(n=333)$} \\
\hline Rule-based system & 0.942 & 0.909 & 0.933 & 0.921 & 0.928 \\
\hline $\mathrm{CNN}$ & 0.994 & 0.994 & 0.994 & 0.994 & 0.994 \\
\hline Logistic regression & 0.906 & 0.865 & 0.896 & 0.877 & 0.888 \\
\hline SVM & 0.864 & 0.894 & 0.917 & 0.830 & 0.877 \\
\hline Random forest & 0.932 & 0.880 & 0.913 & 0.906 & 0.910 \\
\hline
\end{tabular}

${ }^{\mathrm{a}} \mathrm{CNN}$ : convolutional neural network.

${ }^{\mathrm{b}} \mathrm{SVM}$ : support vector machine.

Table 4. Comparison of the neuroimaging interpretation with gold standard and natural language processing.

\begin{tabular}{|c|c|c|c|c|}
\hline $\begin{array}{l}\text { Evaluation of natural language processing against the neuroimaging } \\
\text { interpretation }\end{array}$ & F-measure & kappa & Precision & Recall \\
\hline \multicolumn{5}{|l|}{ Silent brain infarction $(n=24)$} \\
\hline Gold standard & 0.74 & 0.50 & 0.92 & 0.69 \\
\hline $\mathrm{NLP}^{\mathrm{a}}$ & 0.74 & 0.50 & 0.92 & 0.69 \\
\hline \multicolumn{5}{|l|}{ White matter disease $(n=24)$} \\
\hline Gold standard & 0.78 & 0.56 & 0.86 & 0.80 \\
\hline NLP & 0.74 & 0.49 & 0.85 & 0.73 \\
\hline
\end{tabular}

${ }^{\mathrm{a}} \mathrm{NLP}$ : natural language processing. 


\section{Discussion}

\section{Machine Learning Versus Rule}

In summary, the rule-based system achieved the best performance of predicting SBI, and the $\mathrm{CNN}$ model yielded the highest score of predicting WMD. When detecting SBI, the ML models were able to achieve high specificity, NPV, and PPV but moderate sensitivity because of the small number of positive cases. Oversampling is a technique to adjust the class distribution of training data to balance the ratio between positive and negative cases [33]. This technique was applied to the training data to help boost the signals of positive SBIs. The performance was slightly improved but was limited by the issue of overfitting, a situation when a model learns the training data too well. Due to that, unnecessary details and noises in the training data can create negative impact to the generalizability of the model. In our case, the Mayo reports have larger language variation (noise) because of a free style of documentation method, whereas TMC uses a template-based documentation method. According to the sublanguage analysis, Mayo had 212 unique expressions for describing no acute infarction, whereas TMC had only 12. Therefore, the model trained on oversampled data had a bias toward the expressions that only appeared in the training set. When predicting WMD, the ML model outperformed the rule-based model. The reason is because the dataset for WMD is more balanced than SBI (60\% positive cases), which allows the system to equally learn from both classes (positive and negative). The overall performance on WMD is better than SBI because WMDs are often explicitly documented as important findings in the neuroimaging report.

\section{False Prediction Analysis}

Coreference resolution was the major challenge to the rule-based model for identifying SBIs. Coreference resolution is an NLP task to determine whether 2 mentioned concepts refer to the same real-world entity. For example, in Textbox 2, "The above findings" refers to "where there is an associated region of nonenhancing encephalomalacia and linear hemosiderin disposition." To determine if a finding is SBI positive, the system needs to extract both concepts and detect their coreference relationship.

Textbox 2. Example of coreference resolution.

"Scattered, nonspecific T2 foci, most prominently in the left parietal white matter <Concept $1>$ where there is an associated region of nonenhancing encephalomalacia and linear hemosiderin disposition. <Concept 1/> Linear hemosiderin deposition overlying the right temporal lobe (series 9 , image 16) as well. No abnormal enhancement today. <Concept $2>$ The above findings are nonspecific but the evolution, hemosiderin deposition, and gliosis suggest post ischemic change. <Concept $2>$ "

For the ML system, the false positives from the identification of SBIs were commonly contributed by disease locations. As the keywords foci, right occipital lobe, right parietal lobe, right subinsular region, and left frontal region often coexisted with SBI expressions, the model assigned higher weights to these concepts when the model was trained. For example, the expression: "there are a bilateral intraparenchymal foci of susceptibility artifact in the right occipital lobe, right parietal lobe, right subinsular region and left frontal region" has 4 locations with no mention of "infarction" appearing in the sentence. The ML system still predicted it as SBI positive. Among all ML models, the CNN yielded the worse NPV, which suggested the CNN was more likely to receive false signals from disease locations. Our next step is to further refine the system by increasing the volume of training size through leveraging distant supervision to obtain additional SBI positive cases.

\section{Limitations}

Our study has several limitations. First, despite the high feasibility of detecting SBIs from neuroimaging reports, there is a variation between NLP-labeled neuroimaging reports and neuroimages. Second, the performances of the ML models are limited by the number of annotated datasets. Additional training data are required to have a comprehensive comparison between the rule-based and ML systems. Third, the systems were only evaluated using datasets from 2 sites; the generalizability of the systems may be limited.

\section{Conclusions}

We adopted a standardized data abstraction and modeling process to developed NLP techniques (rule-based and ML) to detect incidental SBIs and WMDs from annotated neuroimaging reports. Validation statistics suggested a high feasibility of detecting SBIs and WMDs from EHRs using NLP.

\section{Acknowledgments}

The authors would like to gratefully acknowledge National Institutes of Health grant 1R01NS102233 and Donna M Ihrke for case validation.

\section{Conflicts of Interest}

None declared.

\section{Multimedia Appendix 1}

Gold standard development. 


\section{Multimedia Appendix 2}

Supplementary result.

[PDF File (Adobe PDF File), 465KB-Multimedia Appendix 2]

\section{References}

1. Fanning JP, Wesley AJ, Wong AA, Fraser JF. Emerging spectra of silent brain infarction. Stroke 2014 Nov;45(11):3461-3471. [doi: 10.1161/STROKEAHA.114.005919] [Medline: 25293663]

2. Fanning J, Wong A, Fraser J. The epidemiology of silent brain infarction: a systematic review of population-based cohorts. BMC Med 2014 Jul 9;12:119 [FREE Full text] [doi: 10.1186/s12916-014-0119-0] [Medline: 25012298]

3. Vermeer S, Longstreth JW, Koudstaal P. Silent brain infarcts: a systematic review. Lancet Neurol 2007 Jul;6(7):611-619. [doi: 10.1016/S1474-4422(07)70170-9] [Medline: 17582361]

4. Furie K, Kasner S, Adams R, Albers G, Bush R, Fagan S, American Heart Association Stroke Council, Council on Cardiovascular Nursing, Council on Clinical Cardiology, Interdisciplinary Council on Quality of CareOutcomes Research. Guidelines for the prevention of stroke in patients with stroke or transient ischemic attack: a guideline for healthcare professionals from the american heart association/american stroke association. Stroke 2011 Jan;42(1):227-276. [doi: 10.1161/STR.0b013e3181f7d043] [Medline: 20966421]

5. Gouw A, van der Flier WM, Fazekas F, van Straaten EC, Pantoni L, Poggesi A, LADIS Study Group. Progression of white matter hyperintensities and incidence of new lacunes over a 3-year period: the Leukoaraiosis and Disability study. Stroke 2008 May;39(5):1414-1420. [doi: 10.1161/STROKEAHA.107.498535] [Medline: 18323505]

6. Hijdra A, Verbeeten B, Verhulst J. Relation of leukoaraiosis to lesion type in stroke patients. Stroke 1990 Jun;21(6):890-894. [doi: 10.1161/01.STR.21.6.890] [Medline: 2349592]

7. Miyao S, Takano A, Teramoto J, Takahashi A. Leukoaraiosis in relation to prognosis for patients with lacunar infarction. Stroke 1992 Oct;23(10):1434-1438. [doi: 10.1161/01] [Medline: 1412580]

8. Fu J, Lu C, Hong Z, Dong Q, Luo Y, Wong K. Extent of white matter lesions is related to acute subcortical infarcts and predicts further stroke risk in patients with first ever ischaemic stroke. J Neurol Neurosurg Psychiatry 2005 Jun;76(6):793-796 [FREE Full text] [doi: 10.1136/jnnp.2003.032771] [Medline: 15897500]

9. Chen Y, Wang A, Tang J, Wei D, Li P, Chen K, et al. Association of white matter integrity and cognitive functions in patients with subcortical silent lacunar infarcts. Stroke 2015 Apr;46(4):1123-1126. [doi: 10.1161/STROKEAHA.115.008998] [Medline: 25737316]

10. Conklin J, Silver F, Mikulis D, Mandell D. Are acute infarcts the cause of leukoaraiosis? Brain mapping for 16 consecutive weeks. Ann Neurol 2014 Dec;76(6):899-904. [doi: 10.1002/ana.24285] [Medline: 25283088]

11. Xu H, Jiang M, Oetjens M, Bowton E, Ramirez A, Jeff J, et al. Facilitating pharmacogenetic studies using electronic health records and natural-language processing: a case study of warfarin. J Am Med Inform Assoc 2011;18(4):387-391 [FREE Full text] [doi: 10.1136/amiajnl-2011-000208] [Medline: 21672908]

12. Grishman R, Huttunen S, Yangarber R. Information extraction for enhanced access to disease outbreak reports. J Biomed Inform 2002 Aug;35(4):236-246 [FREE Full text] [doi: 10.1016/S1532-0464(03)00013-3] [Medline: 12755518]

13. South B, Shen S, Jones M, Garvin J, Samore M, Chapman W, et al. Developing a manually annotated clinical document corpus to identify phenotypic information for inflammatory bowel disease. Summit Transl Bioinform 2009 Mar 1;2009:1-32 [FREE Full text] [doi: 10.1186/1471-2105-10-S9-S12] [Medline: 21347157]

14. Gilbert E, Lowenstein S, Koziol-McLain J, Barta D, Steiner J. Chart reviews in emergency medicine research: where are the methods? Ann Emerg Med 1996 Mar;27(3):305-308. [doi: 10.1016/S0196-0644(96)70264-0] [Medline: 8599488]

15. Wang Y, Wang L, Rastegar-Mojarad M, Moon S, Shen F, Afzal N, et al. Clinical information extraction applications: a literature review. J Biomed Inform 2018 Dec;77:34-49 [FREE Full text] [doi: 10.1016/j.jbi.2017.11.011] [Medline: 29162496]

16. Murff H, FitzHenry F, Matheny M, Gentry N, Kotter K, Crimin K, et al. Automated identification of postoperative complications within an electronic medical record using natural language processing. J Am Med Assoc 2011 Aug 24;306(8):848-855. [doi: 10.1001/jama.2011.1204] [Medline: 21862746]

17. Denny J, Ritchie M, Basford M, Pulley J, Bastarache L, Brown-Gentry K, et al. PheWAS: demonstrating the feasibility of a phenome-wide scan to discover gene-disease associations. Bioinformatics 2010 May 1;26(9):1205-1210 [FRE Full text] [doi: 10.1093/bioinformatics/btq126] [Medline: 20335276]

18. Ferrucci D, Lally A. UIMA: an architectural approach to unstructured information processing in the corporate research environment. Nat Lang Eng 1999;10(3-4):327-348. [doi: 10.1017/S1351324904003523]

19. McCarty C, Chisholm R, Chute C, Kullo I, Jarvik G, Larson E, eMERGE Team. The eMERGE Network: a consortium of biorepositories linked to electronic medical records data for conducting genomic studies. BMC Med Genomics 2011 Jan 26;4:13 [FREE Full text] [doi: 10.1186/1755-8794-4-13] [Medline: 21269473]

20. Church K, Hanks P. Word association norms, mutual information, and lexicography. Comput Linguist 1990;16(1):22-29 [FREE Full text]

21. Kim Y. Association for Computational Linguistics. 2014. Convolutional neural networks for sentence classification URL: https://www.aclweb.org/anthology/D14-1181.pdf [accessed 2019-04-08] [WebCite Cache ID 77TSHIa8O] 
22. Abadi M, Barham P, Chen J, Chen Z, Davis A, Dean J, et al. TensorFlow: a system for large-scale machine learning. In: Proceedings of the 12th USENIX conference on Operating Systems Design and Implementation. 2016 Presented at: OSDI'16; November 2-4, 2016; Savannah, GA, USA.

23. Collobert R, Weston J. A unified architecture for natural language processing: deep neural networks with multitask learning. In: Proceedings of the 25th international conference on Machine learning. A unified architecture for natural language processing: Deep neural networks with multitask learning. Proceedings of the 25th international conference on Machine learning; 2008 Presented at: ICML'08; July 5-9, 2008; Helsinki, Finland. [doi: 10.1145/1390156.1390177]

24. Breiman L. Random forests. Mach Learn 2001;45(1):5-32 [FREE Full text] [doi: 10.1023/A:1010933404324]

25. Cortes C, Vapnik V. Support-vector networks. Mach Learn 1995;20(3):273-297 [FREE Full text] [doi: 10.1023/A:1022627411411]

26. Hosmer JD, Lemeshow S, Sturdivant R. Applied Logistic Regression. Hoboken, New Jersey: John Wiley \& Sons; 2013.

27. Wang Y, Liu S, Afzal N, Rastegar-Mojarad M, Wang L, Shen F, et al. A comparison of word embeddings for the biomedical natural language processing. J Biomed Inform 2018 Nov;87:12-20. [doi: 10.1016/j.jbi.2018.09.008] [Medline: 30217670]

28. Mikolov T, Chen K, Corrado G, Dean J. arXiv. 2013. Efficient estimation of word representations in vector space URL: https://arxiv.org/pdf/1301.3781.pdf [accessed 2019-04-08] [WebCite Cache ID 77TWweBoa]

29. Cohen J. A coefficient of agreement for nominal scales. Educ Psychol Meas 2016 Jul 2;20(1):37-46. [doi: $\underline{10.1177 / 001316446002000104]}$

30. McNemar Q. Note on the sampling error of the difference between correlated proportions or percentages. Psychometrika 1947 Jun;12(2):153-157. [doi: 10.1007/BF02295996] [Medline: 20254758]

31. Dietterich T. Approximate statistical tests for comparing supervised classification learning algorithms. Neural Comput 1998;10(7):1895-1923. [doi: 10.1162/089976698300017197]

32. Sasaki Y. Old Dominion University. 2007. The truth of the F-measure URL: https://www.researchgate.net/publication/ 268185911 The truth of the F-measure [accessed 2019-04-08] [WebCite Cache ID 77TXSTW3V]

33. Chawla NV, Bowyer KW, Hall LO, Kegelmeyer WP. arXiv. 2002. SMOTE: synthetic minority over-sampling technique URL: https://arxiv.org/pdf/1106.1813.pdf [accessed 2019-04-08] [WebCite Cache ID 77TXcYm3e]

\title{
Abbreviations \\ CNN: convolutional neural network \\ CT: computed tomography \\ EHR: electronic health record \\ IAA: interannotator agreement \\ IE: information extraction \\ MRI: magnetic resonance imaging \\ NLP: natural language processing \\ NPV: negative predictive value \\ PPV: positive predictive value \\ SBI: silent brain infarction \\ TMC: Tufts Medical Center \\ WMD: white matter disease
}

\author{
Edited by G Eysenbach; submitted 05.09.18; peer-reviewed by J Zheng, V Vydiswaran, D Oram; comments to author 07.01.19; revised \\ version received 26.02.19; accepted 30.03.19; published 21.04.19 \\ Please cite as: \\ Fu S, Leung LY, Wang Y, Raulli AO, Kallmes DF, Kinsman KA, Nelson KB, Clark MS, Luetmer PH, Kingsbury PR, Kent DM, Liu H \\ Natural Language Processing for the Identification of Silent Brain Infarcts From Neuroimaging Reports \\ JMIR Med Inform 2019;7(2):e12109 \\ URL: http://medinform.jmir.org/2019/2/e12109/ \\ doi: $10.2196 / 12109$ \\ PMID: $\underline{31066686}$
}

(C) Sunyang Fu, Lester Y Leung, Yanshan Wang, Anne-Olivia Raulli, David F Kallmes, Kristin A Kinsman, Kristoff B Nelson, Michael S Clark, Patrick H Luetmer, Paul R Kingsbury, David M Kent, Hongfang Liu. Originally published in JMIR Medical Informatics (http://medinform.jmir.org), 21.04.2019. This is an open-access article distributed under the terms of the Creative Commons Attribution License (https://creativecommons.org/licenses/by/4.0/), which permits unrestricted use, distribution, and reproduction in any medium, provided the original work, first published in JMIR Medical Informatics, is properly cited. The 
complete bibliographic information, a link to the original publication on http://medinform.jmir.org/, as well as this copyright and license information must be included. 